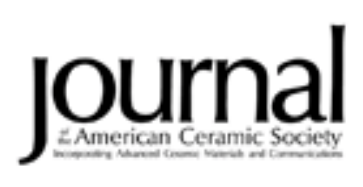

\title{
Inkjet Printing of Zirconia: Coffee Staining and Line Stability
}

\begin{tabular}{|c|c|}
\hline Journal: & Journal of the American Ceramic Society \\
\hline Manuscript ID: & Draft \\
\hline Manuscript Type: & Article \\
\hline $\begin{array}{r}\text { Date Submitted by the } \\
\text { Author: }\end{array}$ & $\mathrm{n} / \mathrm{a}$ \\
\hline Complete List of Authors: & $\begin{array}{l}\text { Dou, Rui; The University of Manchester, Materials Science Centre } \\
\text { Wang, Tianming; University of Manchester, School of Materials } \\
\text { Guo, Yunshan; University of Manchester, School of Materials } \\
\text { Derby, Brian; University of Manchetser, School of Materials }\end{array}$ \\
\hline Keywords: & ink-jet, printing, zirconia, suspensions, drying \\
\hline
\end{tabular}

SCHOLARONE
Manuscripts 


\title{
Inkjet Printing of Zirconia: Coffee Staining
}

\author{
and Line Stability
}

\author{
Rui Dou, Tianming Wang, Yunshan Guo and Brian Derby
}

School of Materials, University of Manchester, Oxford Road, Manchester, M13 9PL, UK

\begin{abstract}
We have prepared a 10 vol \% zirconium oxide powder aqueous ink for inkjet printing. This ink shows severe segregation or coffee staining during drying on solid substrates. Coffee staining is suppressed by the addition of $10 \mathrm{wt} \%$ PEG to the ink formulation. However, coffee staining occurs with the PEG solution ink when drops are printed onto a substrate made from a pre-printed $\mathrm{ZrO}_{2}$ powder substrate. The mechanism for this phenomenon is not known but may be related to the draining of solvent into the powder. The stability of printed features is controlled by the overlap of printed drops and the range of line stability is shown to be consistent with models in the literature.
\end{abstract}




\section{Introduction}

Inkjet printing has great potential in a number of areas of materials manufacture such as: ceramic fabrication, ${ }^{1}$ functional polymers and devices, ${ }^{2}$ biomaterials and tissue engineering. ${ }^{3}$ Inkjet printing is now a mature technology with many years of applications in conventional digital printing and graphics output. However, there is an important distinction between these traditional uses of inkjet printing and its current application in materials fabrication. Digital printing aims to form a pixelated image on a planar substrate. This is achieved by printing individual arrays of dots of dye or pigment that remain isolated as they dry to ensure fidelity of the image. In contrast, materials manufacture normally aims to fabricate continuous features with 1- 2- or 3dimensional connectivity and this is achieved by the interaction of printed drops, often in liquid form, to obtain the desired features. ${ }^{4}$ Hence an important aspect of inkjet printing of ceramics is the interaction of liquid drops deposited on planar surfaces to form stable features prior to drying or solidification of the drops.

In order to fabricate ceramic parts by inkjet printing, an appropriate precursor material must be delivered in a liquid form. Previous studies have shown that fine ceramic powders can be dispersed in an appropriate liquid to make inks suitable for inkjet printing. ${ }^{1,5-8}$ Typically, these inks dry by evaporation and contain about $5-10 \%$ solids by volume. Although it is also possible to use inks that solidify by a temperature induced phase change, ${ }^{9}$ the majority of published work using inkjet printing of ceramic powders has used an evaporating solvent, ${ }^{10}$ mainly because this method leads to high density powder compacts and reduced dimension change during subsequent sintering. However, during the drying of drops containing particles in suspension solute flows may occur and these can result in non-uniform deposition of solid. This 
leads to preferential deposition along the contact line that defines the outer perimeter of the drop. This phenomenon, which is also observed with the deposition of materials from solution, is generally known as coffee staining.

The mechanisms of coffee staining were first explored by Deegan et al, who attributed this phenomenon to the geometrical constraint of the pinned contact line of a drying droplet. ${ }^{11,12}$ The radius of the droplet footprint on the surface does not shrink during dying process because of contact line pinning. Hence the suspension/solution must flow from the droplet centre to perimeter to compensate for evaporative losses. The resulting outward flow can carry virtually all the material to the perimeter, where it is deposited from the solvent to form the ring-like deposit. Under appropriate conditions, coffee staining can result in extreme variations in deposit thickness and this has been exploited to form individual ceramic wells. ${ }^{13}$ In order to produce well defined objects by inkjet printing, it is necessary to control the coffee stain phenomenon and produce homogeneous uniform dried drops. However, it is interesting to note that the contact line pinning, which leads to coffee staining is an important stabilising influence that allows the coalescence of droplets to form well defined surface features. ${ }^{14}$ Thus methods of controlling coffee staining must maintain the stability of liquid deposits prior to drying.

One mechanism by which coffee staining can be controlled is through the use of solvent mixtures. ${ }^{15}$ Evaporation is enhanced at the edge of a drying drop because of the presence of the surrounding region of dry substrate. When a droplet contains two solvents with different evaporation rates, the solvent with the lowest evaporation rate will be enriched at the edge of the droplet as drying progresses. If the solvent mixture 
is selected such that the lower vapour pressure solvent has a smaller surface tension, the concentration gradient resulting from the differential evaporation rates will lead to a gradient in surface tension that generates a flow, known as a Marangoni flow, opposing the flow generated by contact line pinning. The importance of these Marangoni flows during droplet drying can be estimated using the dimensionless Marangoni number, $M a$, defined:

$$
M a=\frac{\Delta \gamma r}{\eta D}
$$

where, $\Delta \gamma$ is the difference in surface tension (taken here to be the difference between the two pure solvents), $r$ is a characteristic length (assumed to be the radius of the spread drop on the substrate), $\eta$ is the fluid viscosity and $D$ is the solute diffusion coefficient. It is generally believed that Marangoni flows are significant if $M a>100$.

Here we present a study of the behaviour of an aqueous $\mathrm{ZrO}_{2}$ ink after inkjet printing. We explore the conditions under which stable individual lines are formed from the coalescence of drops and further investigate whether these are consistent with models developed for the inkjet printing of other fluids. The mechanisms of coffee staining are explored on both solid substrates and when drops are deposited on dried ceramic layers as is needed to produce 3-D objects through sequential overprinting.

\section{Experimental Materials and Methods}

Zirconium oxide powder with specific surface area $=7 \mathrm{~m}^{2} \mathrm{~g}^{-1}$ and mean particle diameter $<200 \mathrm{~nm}$ (99.99\% Tosoh-Zirconia, T2-3YS-E, Tosoh Corporation. Yamagushi, Japan) was used to prepare aqueous inks. A mixture of two different surfactants was chosen to disperse the zirconium oxide powder in water: DISPEX 
A40 (Ciba Specialty Chemicals Inc., Basel, Switzerland), and carboxylic acid prepared DOLAPIX CE 64 (Zshimmier \& Schwarz, Rhein, Germany). The inks were mixed in polyethylene bottles with appropriate quantities of the surfactant by a traditional ball milling process using $\mathrm{ZrO}_{2}$ milling media for up to 7 days. In order to investigate the effect of solvent mixtures on coffee staining, inks were also made with aqueous solvents containing $10 \mathrm{wt} \%$ ethanol or $10 \mathrm{wt} \%$ poly(ethylene glycol) - PEG (BDH, Poole, UK).

Suspension stability was assessed visually by leaving samples in cylindrical glass vessels and examining them for sedimentation at regular intervals. Suspension viscosity was measured using a concentric cylinder rheometer (RSIII, Brookfield, Middleboro, MA, USA). The contact angle (as defined by the Young equation) of the fluid on a range of substrates was determined by sessile drop experiments. Fluid surface tension was measured using a pendant drop method. In both cases the resulting drops were examined using image analysis software (FTA 200, Camtel, Royston, U.K.).

Zirconia suspensions were printed onto clean substrates using an in house designed and built laboratory scale inkjet printing platform that has a positional accuracy of 3 $\mu \mathrm{m}$ (Micromech Systems, Braintree, UK). This is equipped with piezoelectric actuated inkjet printheads of internal diameter $60 \mu \mathrm{m}$ (MJ-ATP-01, Microfab Technologies, Plano, TX, USA) with drive electronics (JetDrive III, Microfab) interfaced to a PC and controlled in a LabVIEW ${ }^{\mathrm{TM}}$ (National Instruments, Austin, TX, USA) environment. Inkjet printing parameters were set at $80 \mathrm{~V}$ peak actuating voltage with $3 \mu$ s rise time, $10 \mu$ s dwell time and $3 \mu$ s fall time at a frequency of $200 \mathrm{~Hz}$, The 
rationale for choosing appropriate printing conditions have been discussed in detail elsewhere. ${ }^{16}$ The dimensions of printed structures were measured either using conventional optical microscopy or with a white light interferometric system (MicroXAM Surface Mapping Microscope, Phase Shift Technology Inc., Tucson, AZ, USA).

Coffee staining behaviour of printed drops was investigated on a range of substrates including: glass microscope slides (BDH, Poole, UK), an epoxy resin (Accura SI 40 material, 3D Systems GmbH, Darmstadt, Germany), pre-printed and dried $\mathrm{ZrO}_{2}$ powder layers. To investigate the stability of printed liquid lines made using the 10 vol $\% \mathrm{ZrO}_{2}$ ink, we printed using the same conditions as with coffee staining tests but with two different nozzle to substrate relative speeds of $40 \mathrm{mms}^{-1}$ and $80 \mathrm{mms}^{-1}$ respectively. We printed lines with droplet spacing values in the range from $10 \mu \mathrm{m}$ to $80 \mu \mathrm{m}$ to study the morphological stability of the resulting printed lines.

In order to study the stability of drops printed on top of a dried printed ceramic powder layer, a single ceramic layer, was prepared on an epoxy resin substrate by inkjet printing a pattern of overlapping drops. Subsequently individual isolated dots and lines were printed onto the pre-printed layer to allow the study of coffee staining during multi-layer printing. 


\section{Results and Discussion}

\section{Ink preparation}

To optimize the composition of the $\mathrm{ZrO}_{2}$ aqueous ink, we have measured the viscosities of $10 \mathrm{vol} \%$ suspensions using a range of surfactant dosage. A contour map of the suspensions' viscosity is shown in Figure 1. The viscosity of the ink increases with increasing dosage of surfactant, especially with DOLAPIX CE 64. The optimised composition for the ink is $10 \mathrm{vol} \% \mathrm{ZrO}_{2}$ powder aqueous suspensions with 1 wt $\%$ DISPEX A 40 and 0.5 wt \% DOLAPIX CE 64 . Note that the viscosity values plotted in Figure 1 were taken from samples after identical milling time, but are not the final viscosities of the optimized suspensions. After efficient milling (7 days), the optimized $\mathrm{ZrO}_{2}$ ink had a viscosity of $2.46 \mathrm{mPas}$ and was suitable for inkjet printing. Sedimentation tests on the inks indicate that the inks were stable over time intervals consistent with printing experiments. After standing for $24 \mathrm{~h}$, there is no visible sedimentation with only 4 vol \% upper clear water in the ink.

\section{Segregation during drying (Coffee Staining)}

Figure 2 shows 3-D reconstruction images obtained from interferometric microscopy data of single droplets of the 10 vol $\% \mathrm{ZrO}_{2}$ ink inkjet printed onto glass substrates and dried. The temperature range of the substrates applied in this study is from $25^{\circ} \mathrm{C}$ to $200{ }^{\circ} \mathrm{C}$. At all temperatures there is significant coffee staining with most of the $\mathrm{ZrO}_{2}$ particles depositing towards the edge of the drop. The diameter of the dried droplet is seen to decrease dramatically when the substrate temperature is increased to $35{ }^{\circ} \mathrm{C}$, but shows little subsequent reduction in radius with further increase in 
temperature. The height difference between the droplet edge and centre is found to increase with increasing substrate temperature. It has been observed in other work on droplet drying that printing onto a heated substrate can result in a reduction in coffee staining. ${ }^{17,18}$ However, in this case an increase in temperature has little influence on coffee staining, which is consistent with the proposal of Soltman and Subramaian ${ }^{19}$ and the modelling work of Ozawa ${ }^{20}$ that the heating of the substrate will preferentially enhance evaporation close to the contact line and thus increase the driving force for coffee staining.

In order to investigate the influence of solvent mixtures on coffee staining the aqueous solvent was mixed with $10 \mathrm{wt} \%$ ethanol or PEG prior to the addition of surfactants and $\mathrm{ZrO}_{2}$. Both ethanol and PEG reduce the surface tension of water with increasing concentration but ethanol/water mixtures will preferentially lose ethanol through evaporation while PEG/water mixtures will lose water. In which case, we expect the influence of differential evaporation to lead to a Marangoni flow that opposes coffee staining with the PEG/water solvent mixture and a flow that does not impede coffee staining with ethanol/water mixtures. Figure 3 and 4 shows results from dried drops obtained from these solvent mixtures after inkjet printing onto glasss substrates at a range of temperatures.

The behaviour of dried drops obtained using ethanol/water mixtures are shown in Figure 3. Coffee staining is still present at all substrate temperatures, however, there are significant differences between the behaviour of this mixture and that of pure water suspensions (Figure 2). The diameter of the dried drops shows less variation with temperature and the width of the deposit at the contact line is increased and its 
height is reduced. This reduction in the severity of the segregation may indicate that the contact line is less well pinned than with the aqueous suspensions and that some retraction of the contact line has occurred.

The behaviour of drops printed using the PEG/water suspensions are shown in Figure 4. At 25 and $35^{\circ} \mathrm{C}$ (Figures $4 \mathrm{a}$ and $4 \mathrm{~b}$ ) coffee staining has been eliminated, however, at higher substrate temperatures coffee staining and segregation of the dried deposit is seen (Figures $4 \mathrm{c}$ and $4 \mathrm{~d}$ ). This behaviour is consistent with the expected behaviour of the Marangoni effect as quantified by the Marangoni number (Equation 1). Coffee staining is opposed by a surface tension driven flow set up by a concentration gradient. Diffusion within the liquid droplet will tend to reduce the concentration gradient and thus increasing temperature will decrease the value of the Marangoni number because of the presence of the diffusion coefficient in the denominator of Equation 1. However at room temperature the addition of PEG to the aqueous solution is sufficient to prevent coffee staining.

\section{Stability of Printed Surface Features}

Solid objects are produced by the sequential printing of layers. Each layer is formed by a number of overlapping liquid drops that coalesce to form the desired pattern. These drops remain on the surface in liquid form until they dry, hence there is a period of time when there will be a series of 2-D liquid features that must remain stable and maintain their desired shape for a period of time on the surface. The stability of liquid sheets has not been researched in great detail. However, there has been significant work on the stability of linear liquid features, such as are formed by the coalescence of a line of overlapping drops. Davis proposed that liquid linear 
features or beads can be stable on a smooth surface if the contact line is pinned ${ }^{21}$ and this was confirmed experimentally by Schiaffino and Sonin. ${ }^{22}$ Smith et al identified a maximum drop spacing, below that for droplet overlap, above which drops do not form parallel sided liquid tracks. ${ }^{23}$ Duineveld observed a minimum drop spacing below which a dynamic instability set in, which results in regular spaced bulges along a liquid track. ${ }^{24}$ These two instabilities were used by Soltman and Subramanian to propose that there is a limited set of drop spacings between which stable parallel side lines can be formed by inkjet printing. ${ }^{19}$

The limit set by the maximum spacing of overlapping drops is shown to be related to the equilibrium behavior of spreading liquid limited by contact line pinning. ${ }^{23}$ The minimum drop spacing below which the bulging instability occurs was shown by Duineveld to be a dynamic function of the transverse velocity of the printing device relative to the substrate, $U_{T} \cdot{ }^{24}$ In dimensionless form this velocity is

$$
U_{T}^{*}=\frac{\eta U_{T}}{\sigma_{L V}}
$$

where $\eta$ is the dynamic viscosity of the fluid and $\sigma_{L V}$ is the liquid/vapor interfacial energy. Stringer developed Duineveld's model ${ }^{14}$ to show that stable liquid tracks are formed if the dimensionless velocity is greater than a function of the dimensionless drop spacing, $p^{*}$, and the advancing contact angle of the liquid on the substrate, $\theta_{a}$.

$$
U_{T}^{*}>g\left(p^{*}, \theta_{a}\right)
$$

with 


$$
p^{*}=\frac{p}{\beta_{e q m} d_{0}}
$$

where $p$ is the spacing of drops on the substrate, $d_{0}$ is the diameter of the original spherical drop and $\beta_{\text {eqm }}=d_{\text {eqm }} / d_{0}$ or the ratio between the equilibrium spread diameter of the drop on the substrate, $d_{e q m}$, and $d_{\mathrm{o}}{ }^{14}$

Stringer et al showed that the function $g\left(p^{*}, \theta\right)$ is related to the inverse of the drop spacing and the contact angle and is given explicitly as Equation 16c in ref. [14]. The fluid property data required for the model is presented in Table 1. A number of tracks were printed with varying amounts of droplet overlap to explore the validity of the model. Two nozzle to substrate transverse speeds $\left(U_{T}\right)$ of $40 \mathrm{mms}^{-1}$ and $80 \mathrm{mms}^{-1}$ were used to investigate printed line stability over a range of drop spacing $p$ in the range $10 \mu \mathrm{m}$ to $100 \mu \mathrm{m}$.

Figure 5 shows optical microscope images of printed lines from both the original aqueous ink and a $10 \mathrm{wt} \%$ PEG solution ink. Both inks show similar behavior with parallel sided tracks printed at a drop spacing of $50 \mu \mathrm{m}$. However, at low drop spacing $(30 \mu \mathrm{m})$ a bulging instability was seen (Figures 5a and 5d) and at large drop spacings $(90 \mu \mathrm{m})$ there is insufficient droplet overlap for a stable parallel sided track to form. This behaviour is qualitatively the same as that observed by Soltman and Subramanian for solutions of PEDOT/PSS ${ }^{19}$ and by Stringer et al with a silvernanoparticle ink. ${ }^{14}$ Note that lines printed using the aqueous ink in Figure $5 \mathrm{a}, \mathrm{b}$ and c show coffee staining as raised ridges at their edges, while the lines printed with the 10 wt $\%$ PEG ink show no effect. Figure 6 shows the predictions of the model derived by Stringer et al for the onset of the bulging instability, ${ }^{14}$ our experimental 
results show excellent agreement.

\section{Multilayer Printing}

The fabrication of 3-D objects can be achieved by printing second and subsequent layers on top of pre-printed and dried layers. Figure 7 shows single pass printed dots and lines using the $10 \mathrm{wt} \%$ PEG ink on an epoxy resin substrate and on a pre-printed layer of dried $\mathrm{ZrO}_{2}$ powder. The printed objects show no coffee staining on the epoxy resin substrate, but the dots and lines on the pre-printed layer show obvious coffee staining. In both cases the printed features were dried in air at room temperature (approximately $25{ }^{\circ} \mathrm{C}$ ). Figure 8 shows reconstructed 3-D images and line profiles across printed dot features made from single drops and repeated overprints of 5 and 10 drops on both the epoxy resin and the $\mathrm{ZrO}_{2}$ powder surfaces. In all cases the drops printed on the epoxy resin substrate show a single central peak and dry to form a convex mound, consistent with our previous observation of isolated drops and printed lines. However, when the ink is printed on top of the $\mathrm{ZrO}_{2}$ powder that had been previously printed, severe coffee staining occurs.

To the best of our knowledge, this is the first report of coffee staining during drop drying on porous substrates. All prior work on coffee staining has considered solvent removal by evaporation and the resulting flows that occur if the contact line is pinned. Because of this focus on evaporation, methods to reduce coffee staining have concentrated on modifying the loss of solvent by evaporation and generating countervailing Marangoni flows. The choice of PEG solutions to reduce coffee staining in this work was guided by these principles and prior work in the literature. Thus we hypothesise that the unexpected appearance of coffee staining after printing 


\section{Conclusions}

We have prepared 10 vol $\% \mathrm{ZrO}_{2}$ aqueous suspensions that have low viscosity and sufficient stability to be suitable for inkjet printing. However, when individual drops and simple linear features are printed, they show significant solute redistribution during drying, also known as coffee staining. This coffee staining can be removed on solid substrates using appropriate solvent mixtures, which was achieved in this case by adding $10 \mathrm{wt} \%$ PEG into the original ink. Using the modified ink, coffee staining was completely eliminated at room temperature $\left(25^{\circ} \mathrm{C}\right)$, however, at temperatures above $35{ }^{\circ} \mathrm{C}$ coffee staining was again observed and this can be explained by diffusion reducing the concentration gradients that drive Marangoni flow. When material was printed onto a layer of dried ceramic powder, coffee staining was found at room temperature with the PEG modified ink. This is believed to be because in this case the drops dry by the draining of fluid into the dried powder bed. This phenomenon may have implications for other applications of printing onto porous substrates as well as for ceramic processing. 
We have also studied the formation of linear features by overlapping printed drops. Stable parallel sided lines are formed within a limited range of drop spacing as has been reported in the literature for other printed fluids. The minimum drop spacing, below which a bulging instability is seen, is found to consistent with the predictions of Stringer's model. ${ }^{14}$

\section{References}

1) B. Derby and N. Reis, "Inkjet printing of highly loaded particulate suspensions," MRS Bulletin, 28 [11] 815-818 (2003).

2) P. Calvert, "Inkjet printing for materials and devices," Chem. Mater., 13 [10] 32993305 (2001).

3) B. Derby, "Bioprinting: inkjet printing proteins and hybrid cell-containing materials and structures," J. Mater. Chem., 18 [47] 5717-5721 (2008).

4) B. Derby, "Inkjet printing of functional and structural materials - fluid property requirements, feature stability and resolution", Ann. Rev. Mater. Res. 40, 395-414 (2010).

5) W. D. Teng, M. J. Edirisinghe, and J. R. G. Evans, "Optimization of dispersion and viscosity of a ceramic let printing ink," J. Amer. Ceram, Soc., 80 [2] 486-494 (1997).

6) Q. F. Xiang, J. R. G. Evans, M. J. Edirisinghe, and P. F. Blazdell, "Solid freeforming of ceramics using a drop-on-demand jet printer," Proceedings of the Institution of Mechanical Engineers Part B-Journal of Engineering Manufacture, 211 [3] 211-214 (1997).

7) C. E. Slade and J. R. G. Evans, "Freeforming ceramics using a thermal jet printer," Journal of Materials Science Letters, 17 [19] 1669-1671 (1998). 
8) M. Mott, J. H. Song, and J. R. G. Evans, "Microengineering of ceramics by direct ink-Jet printing," Journal of the American Ceramic Society, 82 [7] 1653-1658 (1999).

9) K.A.M. Seerden, N. Reis, J.R.G. Evans, P.S. Grant, J.W. Halloran and B. Derby, “Inkjet Printing of Wax Based Ceramic Suspensions", J. Amer. Ceram. Soc. 84 [11] 2514-2520 (2001).

10) B. Derby, "Inkjet Printing Ceramics: From Drops to Solid", J. Europ. Ceram. Soc. In press (2011) doi:10.1016/j.jeurceramsoc.2011.01.016

11) R. D. Deegan, O. Bakajin, T. F. Dupont, G. Huber, S. R. Nagel, and T. A. Witten, "Capillary flow as the cause of ring stains from dried liquid drops," Nature, 389827 829 (1997).

12) R. D. Deegan, O. Bakajin, T. F. Dupont, G. Huber, S. R. Nagel, and T. A. Witten, “Contact line deposits in an evaporating drop", Phys. Rev. E 62 [1] 756-65 (2000)..

13) Y. Zhang, L. F. Chen, S. F. Yang, and J. R. G. Evans, "Preparation of ceramic well plates for combinatorial methods using the morphogenic effects of droplet drying," J. Amer. Ceram. Soc. 89 [12] 3858-3860 (2006).

14) J. Stringer and B. Derby, "Formation and Stability of Lines Produced by Inkjet Printing," Langmuir, 26 [12] 10365-10372 (2010).

15) B. J. de Gans and U. S. Schubert, "Inkjet printing of well-defined polymer dots and arrays". Langmuir 20 [18] 7789-93 (2004).

16) N. Reis, C. Ainsley, and B. Derby, "Inkjet delivery of particle suspensions by piezoelectric droplet ejectors", J. Appl. Phys. 97, 094903 (2005).

17) J. W. Song, J. Kim, Y. H. Yoon, B. S. Choi, J. H. Kim and C. S. Han, "Inkjet printing of single-walled carbon nanotubes and electrical characterization of the line pattern" Nanotechnology 19 [9] 095702 (2008). 
18) T. Wang, M. A. Roberts, I. A. Kinloch and B. Derby, "Inkjet Printed Carbon Nanotube Networks: The Influence of Drop Spacing and Drying on Electrical Properties", submitted to Nanotechnology (2011).

19) D. Soltman and V. Subramanian, "Inkjet-printed line morphologies and temperature control of the coffee ring effect", Langmuir 24 [5] 2224-31 (2008).

20) K. Ozawa, E. Nishitani and M. Doi "Modelling of the drying process of liquid droplet to form thin film", Japanese Journal of Applied Physics Part 144 [6A] 422934 (2005).

21) S. H. Davis, "Moving contact lines and rivulet instabilities. 1. The static rivulet". J Fluid Mech 98 [May] 225-42 (1980).

22) S. Schiaffino and A. A. Sonin. "Formation and stability of liquid and molten beads on a solid surface". J Fluid Mech 343 [July] 95-110 (1997).

23) P. J. Smith, D. Y. Shin, J.E. Stringer, B. Derby and N. Reis. "Direct ink-jet printing and low temperature conversion of conductive silver patterns". J Mater Sci 41 [13] 4153-8 (2006).

24) P. C. Duineveld, “The stability of ink-jet printed lines of liquid with zero receding contact angle on a homogeneous substrate." J. Fluid Mech. 477 [Feb] 175-200 (2003). 


\section{Figure and Table Captions}

Table 1. Physical properties of the printed inks required for the bulging instability model.

Figure 1. Viscosity contour map of the 10 vol $\%$ zirconium oxide powder aqueous ink with two different surfactants.

Figure 2. Interferometric microscope images of dried single droplets printed by using the 10 vol \% zirconium oxide powder aqueous ink on glass substrates at different temperatures: (a) $25^{\circ} \mathrm{C}$; (b) $35^{\circ} \mathrm{C}$; (c) $50^{\circ} \mathrm{C}$; (d) $75^{\circ} \mathrm{C}$; (e) $100^{\circ} \mathrm{C}$; (f) $200^{\circ} \mathrm{C}$.

Figure 3. Interferometric microscope images of dried single droplets printed by using the 10 vol $\%$ zirconium oxide powder aqueous ink with $10 \mathrm{wt} \%$ ethanol on glass substrates at different temperatures: (a) $25^{\circ} \mathrm{C}$; (b) $35^{\circ} \mathrm{C}$; (c) $50^{\circ} \mathrm{C}$; (d) $100^{\circ} \mathrm{C}$.

Figure 4. Interferometric microscope images of dried single droplets printed by using the $10 \mathrm{vol} \%$ zirconium oxide powder aqueous ink with $10 \mathrm{wt} \%$ polyethylene glycol on glass substrates at different temperatures: (a) $25^{\circ} \mathrm{C}$; (b) $35^{\circ} \mathrm{C}$; (c) $50^{\circ} \mathrm{C}$; (d) $100^{\circ} \mathrm{C}$. 
Figure 5. Optical microscope images of dried lines printed by using the 10 vol $\%$ $\mathrm{ZrO}_{2}$ aqueous ink $(\mathrm{a}, \mathrm{b}, \mathrm{c})$ and the ink with $10 \mathrm{wt} \%$ PEG (d, e, f) on epoxy resin substrates: (a) $p=30 \mu \mathrm{m}, U_{T}=40 \mathrm{mms}^{-1}$; (b) $p=50 \mu \mathrm{m}, U_{T}=40 \mathrm{mms}^{-1}$; (c) $p=$ $90 \mu \mathrm{m}, U_{T}=40 \mathrm{mms}^{-1}$; (d) $p=30 \mu \mathrm{m}, U_{T}=40 \mathrm{mms}^{-1}$; (e) $p=50 \mu \mathrm{m}, U_{T}=40$ $\mathrm{mms}^{-1}$; (f) $p=90 \mu \mathrm{m}, U_{T}=40 \mathrm{mms}^{-1}$.

Figure 6. Stability map showing the condition for the onset of bulging instability, with data from experiments on our 10 vol $\%$ zirconium oxide powder aqueous ink superimposed, together with data from reference ${ }^{10}$. In all cases, open symbols indicate unstable morphology and filled symbols indicate stable morphology.

Figure 7. Optical microscope images of dots and lines on the epoxy resin substrate and pre-printed layer, which are printed by using the original ink with $10 \mathrm{wt} \%$ PEG: (a) a dot on epoxy resin substrate; (b) a dot on the pre-printed layer; (c) a line on epoxy resin substrate; (d) a line on the pre-printed layer.

Figure 8. Interferometric microscope images of dried dots printed by using the 10 vol \% original ink with $10 \mathrm{wt} \%$ PEG by single and multi-passes printing on epoxy resin substrate (left row) and the pre-printed layer (right row). 


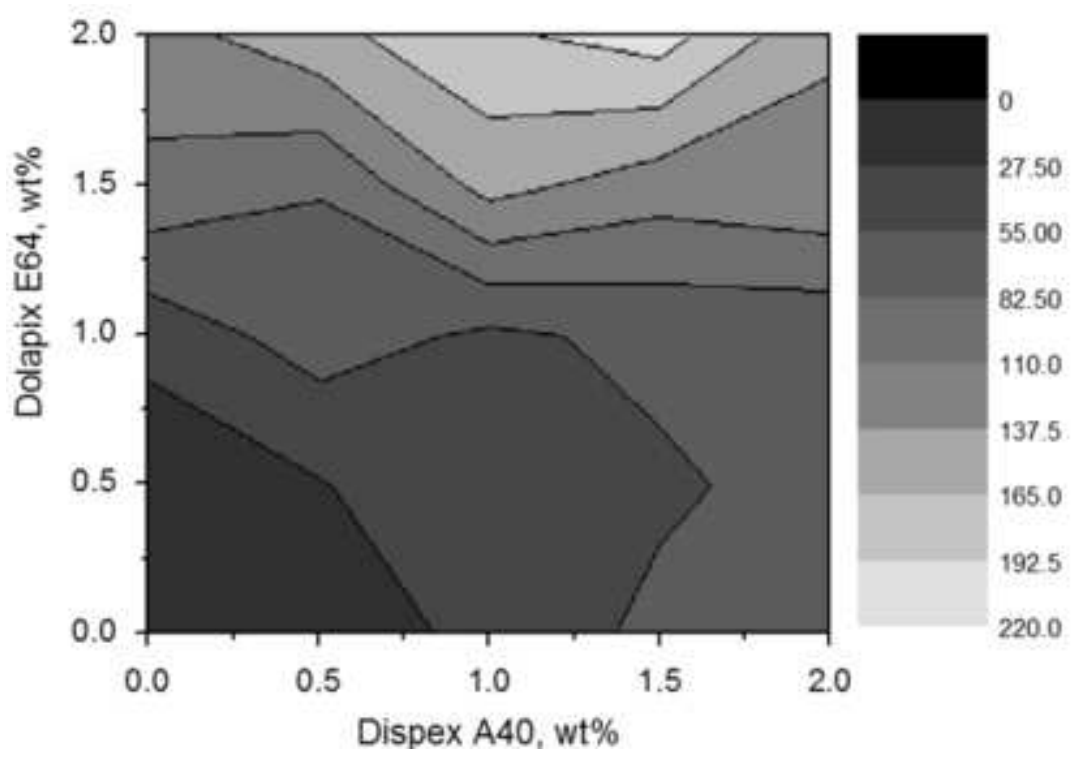

Figure 1 . Viscosity contour map of the $10 \mathrm{vol} \%$ zirconium oxide powder aqueous ink with two different surfactants. $17 \times 12 \mathrm{~mm}(600 \times 600 \mathrm{DPI})$ 


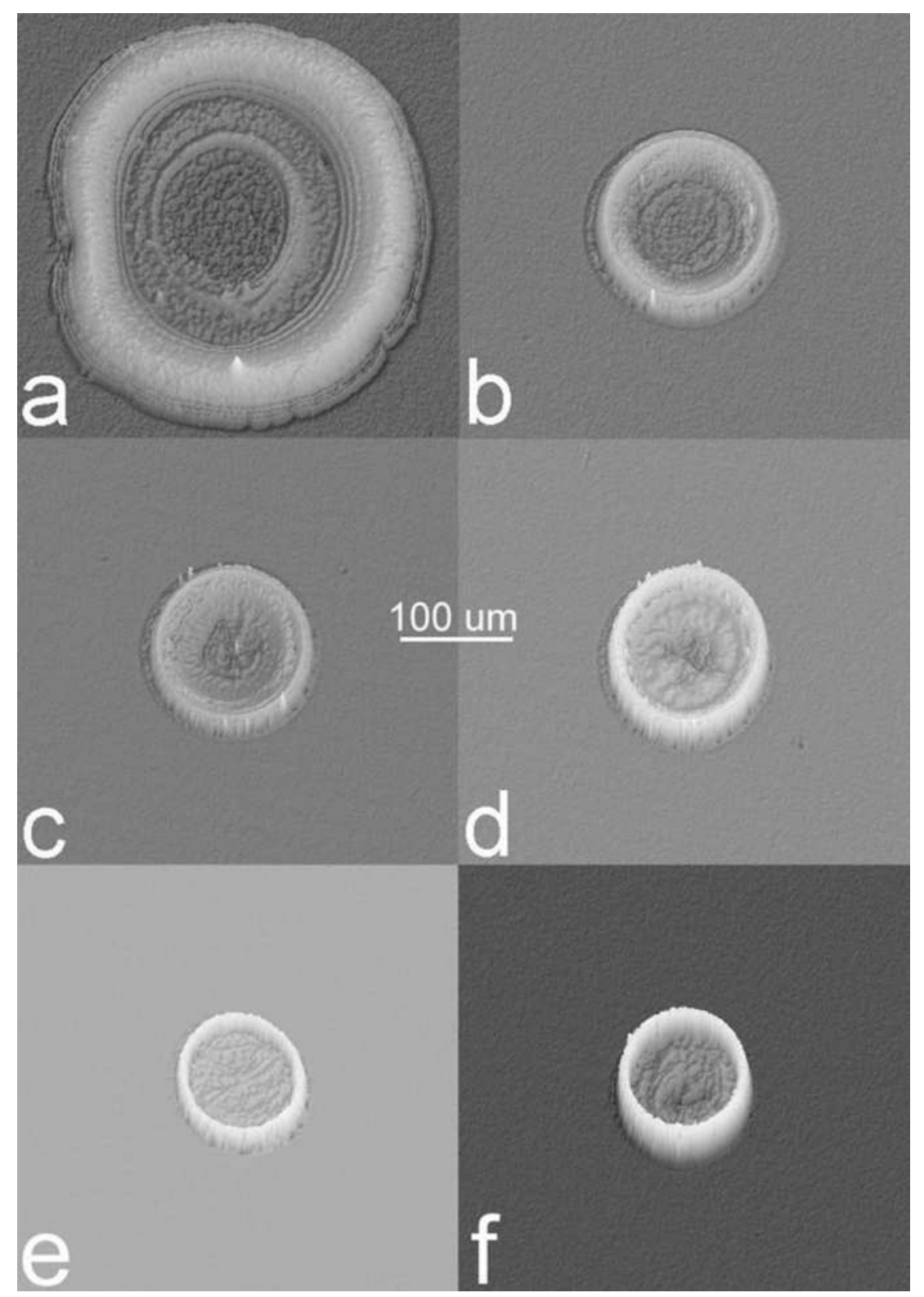

Figure 2. Interferometric microscope images of dried single droplets printed by using the $10 \mathrm{vol} \%$ zirconium oxide powder aqueous ink on glass substrates at different temperatures: (a) $25^{\circ} \mathrm{C}$; (b) $35^{\circ} \mathrm{C}$; (c) $50^{\circ} \mathrm{C}$; (d) $75^{\circ} \mathrm{C}$; (e) $100^{\circ} \mathrm{C}$; (f) $200^{\circ} \mathrm{C}$. $28 \times 40 \mathrm{~mm}(600 \times 600 \mathrm{DPI})$ 


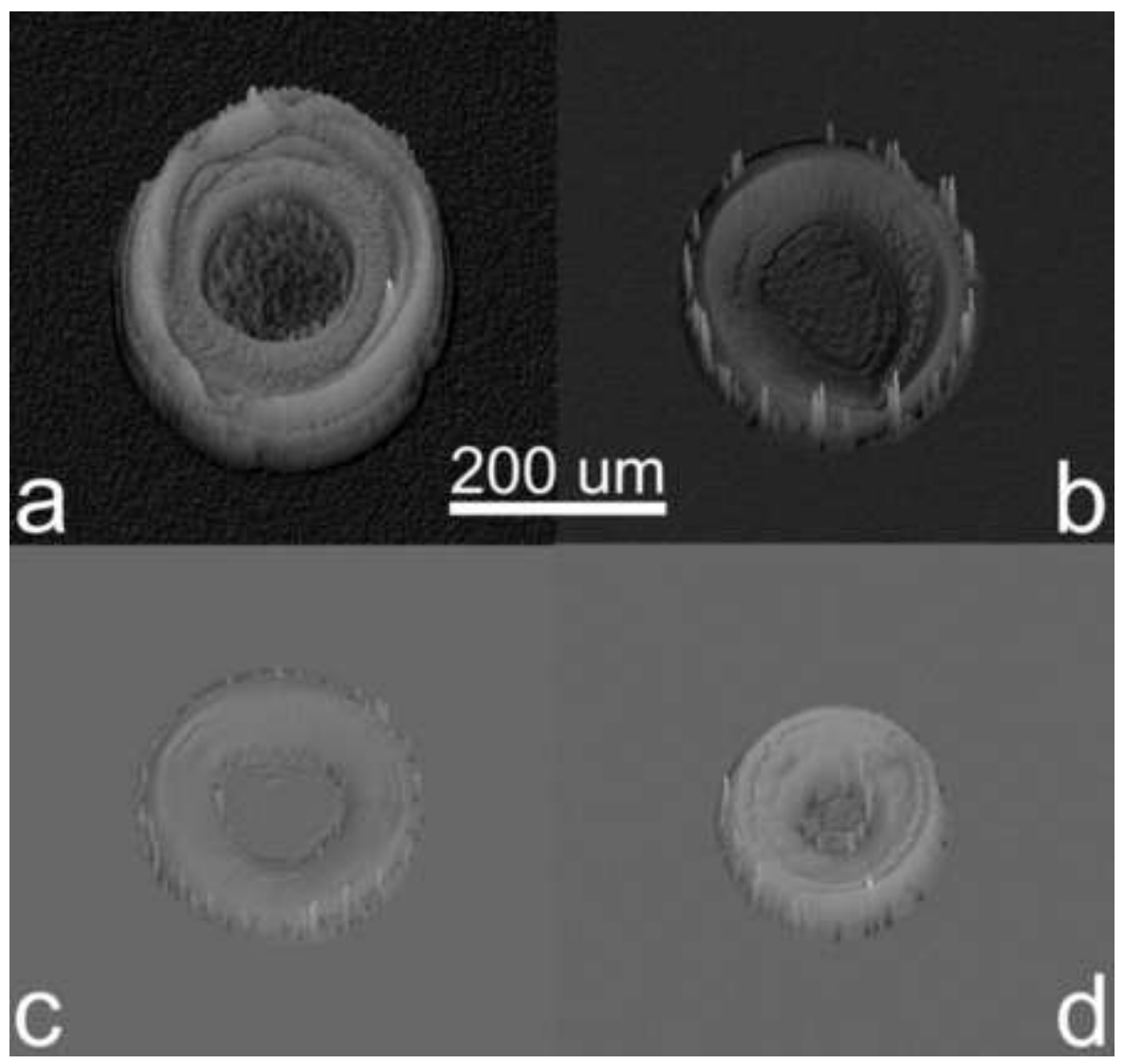

Figure 3. Interferometric microscope images of dried single droplets printed by using the 10 vol \% zirconium oxide powder aqueous ink with $10 \mathrm{wt} \%$ ethanol on glass substrates at different temperatures: (a) $25^{\circ} \mathrm{C}$; (b) $35^{\circ} \mathrm{C}$; (c) $50^{\circ} \mathrm{C}$; (d) $100^{\circ} \mathrm{C}$.

$18 \times 17 \mathrm{~mm}(600 \times 600 \mathrm{DPI})$ 


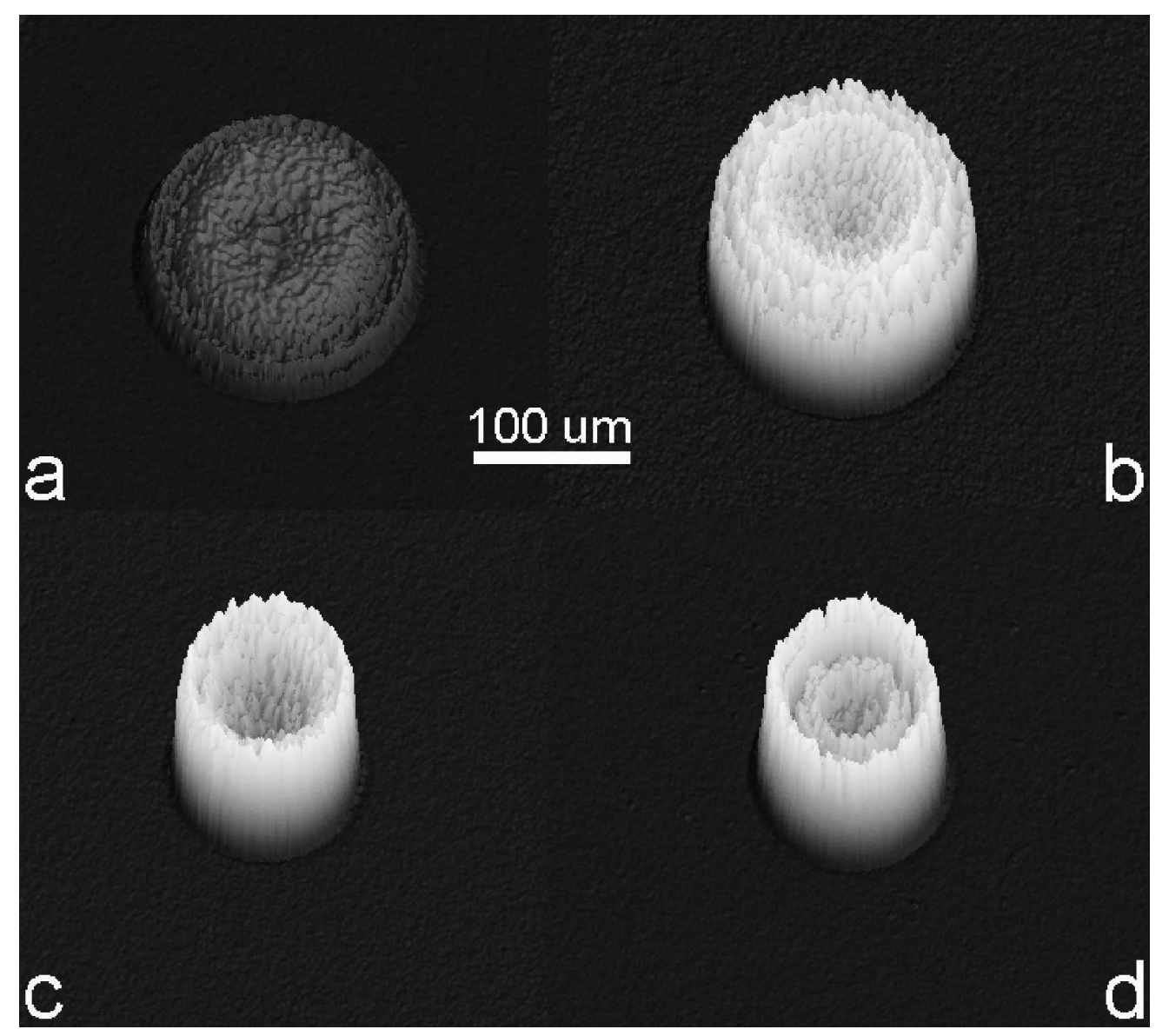

Figure 4. Interferometric microscope images of dried single droplets printed by using the $10 \mathrm{vol} \%$ zirconium oxide powder aqueous ink with $10 \mathrm{wt} \%$ polyethylene glycol on glass substrates at different temperatures: (a) $25^{\circ} \mathrm{C}$; (b) $35^{\circ} \mathrm{C}$; (c) $50^{\circ} \mathrm{C}$; (d) $100^{\circ} \mathrm{C}$. 


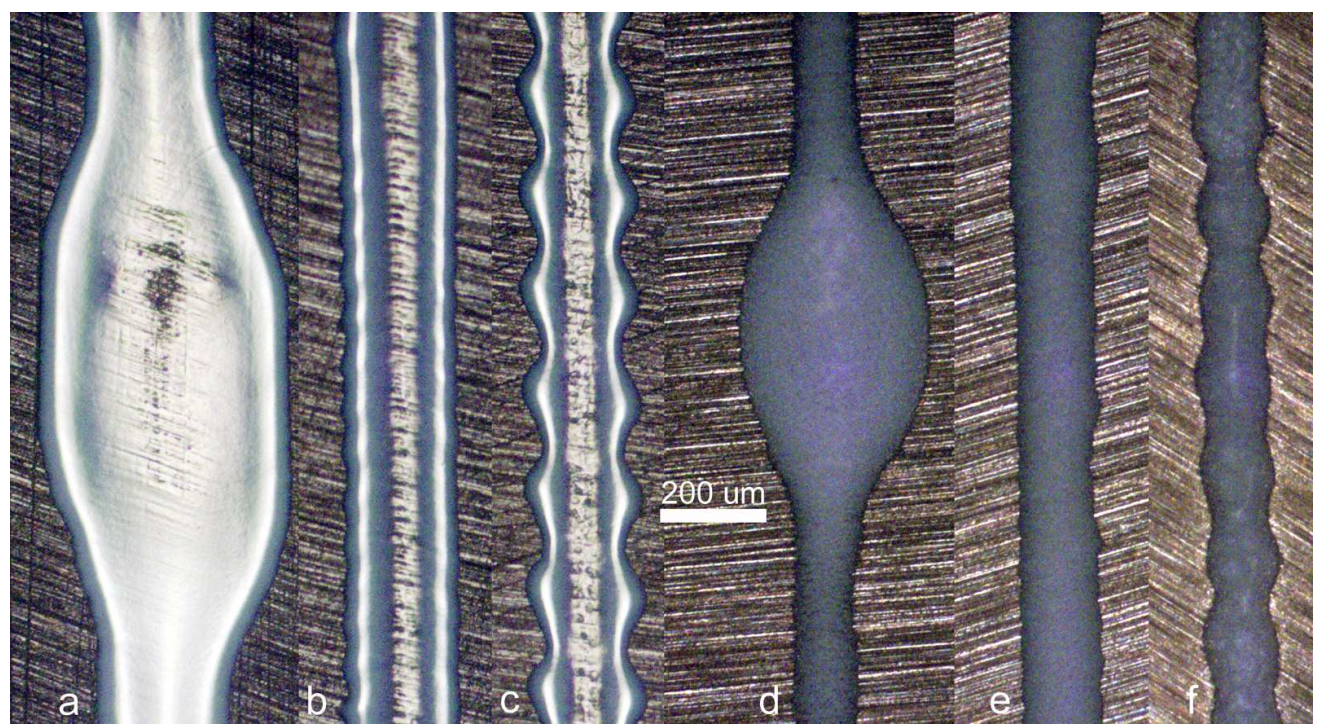

Figure 5. Optical microscope images of dried lines printed by using the $10 \mathrm{vol} \% \mathrm{ZrO} 2$ aqueous ink $(a, b, c)$ and the ink with 10 wt \% PEG $(d, e, f)$ on epoxy resin substrates: $(a)=30 \mu m,=40$ $\mathrm{mms}-1 ;(\mathrm{b})=50 \mu \mathrm{m},=40 \mathrm{mms}-1 ;(\mathrm{c})=90 \mu \mathrm{m},=40 \mathrm{mms}-1 ;(\mathrm{d})=30 \mu \mathrm{m},=40 \mathrm{mms}-1$; (e) $=50 \mu \mathrm{m},=40 \mathrm{mms}-1 ;(\mathrm{f})=90 \mu \mathrm{m},=40 \mathrm{mms}-1$. $67 \times 36 \mathrm{~mm}(600 \times 600 \mathrm{DPI})$ 


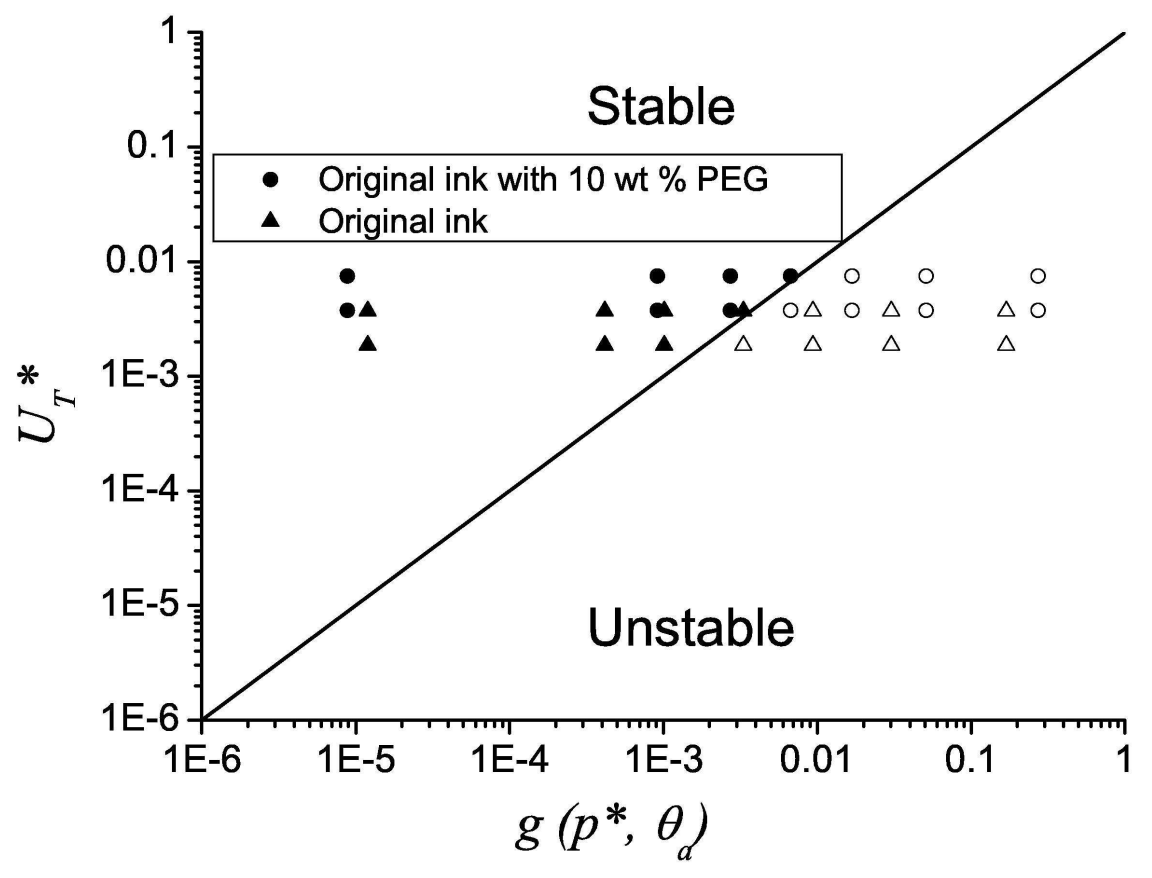

Figure 6. Stability map showing the condition for the onset of bulging instability, with data from experiments on our $10 \mathrm{vol} \%$ zirconium oxide powder aqueous ink superimposed, together with data from reference10. In all cases, open symbols indicate unstable morphology and filled symbols indicate stable morphology. $114 \times 87 \mathrm{~mm}(600 \times 600 \mathrm{DPI})$ 
Figure 7. Optical microscope images of dots and lines on the epoxy resin substrate and pre-printed layer, which are printed by using the original ink with $10 \mathrm{wt} \%$ PEG: (a) a dot on epoxy resin substrate; (b) a dot on the pre-printed layer; (c) a line on epoxy resin substrate; (d) a line on the pre-printed layer. $28 \times 24 \mathrm{~mm}(600 \times 600 \mathrm{DPI})$ 


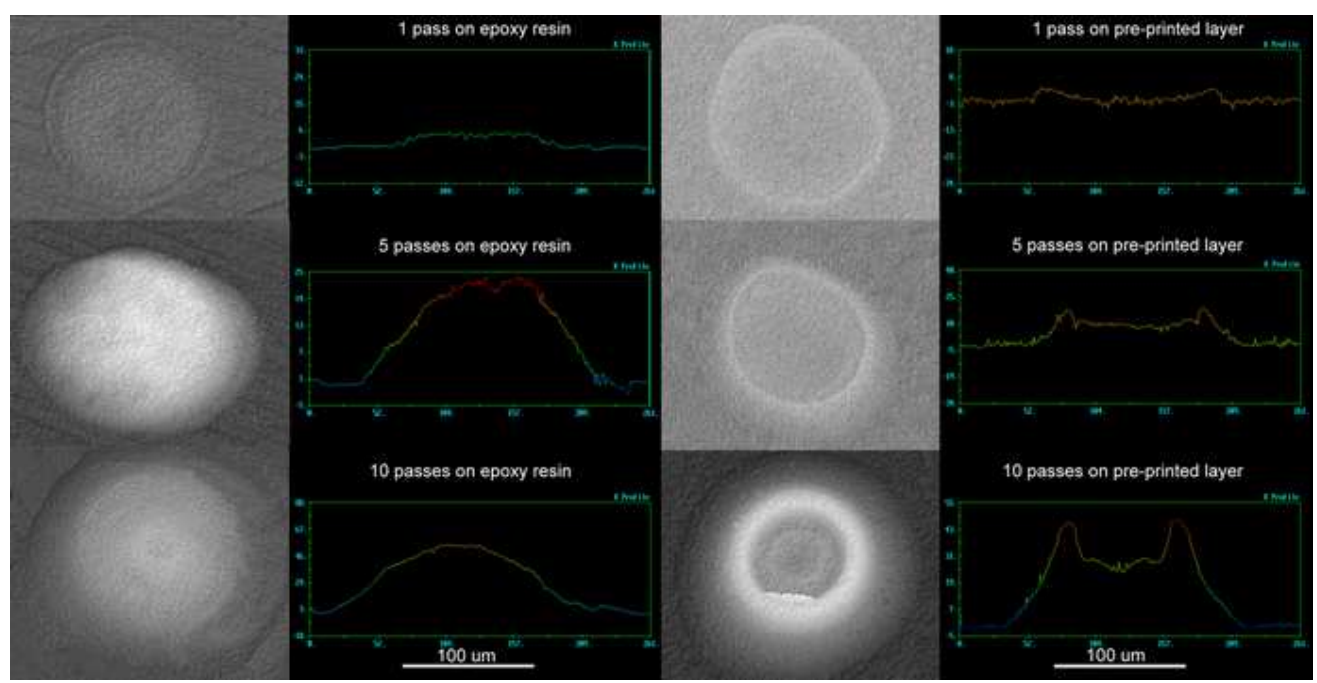

Figure 8. Interferometric microscope images of dried dots printed by using the 10 vol \% original ink with 10 wt \% PEG by single and multi-passes printing on epoxy resin substrate (left row) and the pre-printed layer (right row). $29 \times 14 \mathrm{~mm}(600 \times 600 \mathrm{DPI})$ 
Table 1. Physical properties of the printed inks required for the bulging instability model.

\begin{tabular}{lcccc}
\hline Ink & $\boldsymbol{\theta}$ & $\boldsymbol{\theta}_{\boldsymbol{a}}$ & $\boldsymbol{\sigma}_{\boldsymbol{L} V}\left(\mathbf{m J m}^{-2}\right)$ & $\boldsymbol{\eta}(\mathbf{m P a s})$ \\
\hline $10 \mathrm{vol} \% \mathrm{ZrO}_{2}$ & $56 \pm 2.8^{\circ}$ & $50 \pm 1.2^{\circ}$ & 53 & 2.46 \\
$10 \mathrm{vol} \% \mathrm{ZrO}_{2} / \mathrm{PEG}$ & $60 \pm 2.6^{\circ}$ & $53 \pm 1.8^{\circ}$ & 48 & 4.51 \\
\hline
\end{tabular}

